\title{
Behavioral problems as a risk factor for Alopecia Areata and another skin disease: a case-control study in Iran
}

Najmeh shahini ( $\sim$ Najmeh.shahini@gmail.com )

Golestan University of Medical Sciences

Firoozeh Derakhshanpour

Golestan University of Medical Sciences

zanireh salami

Mashhad University of Medical Sciences

kimia kazemian

Lorestan University of Medical Sciences

GholamReza Vaghari

Golestan Golestan University of Medical Sciences

\section{Research Article}

Keywords: Behavioral problems, alopecia areata, pediatrics

Posted Date: March 2nd, 2022

DOI: https://doi.org/10.21203/rs.3.rs-1391290/v1

License: (c) (i) This work is licensed under a Creative Commons Attribution 4.0 International License. Read Full License 


\section{Abstract}

Background: Alopecia areata is a dermatological disorder that is more prevalent among the pediatric population. This disease has been linked to behavioral problems in both pediatrics and adults. However, the behavioral problems of the pediatric population have not been widely studied. The present study aimed to evaluate the behavioral problems among the pediatric population in the north of Iran.

methods: This case-control study was carried out on the 90 children aged 4-13 who have been chosen by simple sampling and equally enrolled in three study groups; Alopecia areata, another skin disease, and healthy children. The parent's version of the strengths and difficulties questionnaire (SDQ) was used, and $\mathrm{P}<0.05$ is considered a significant level.

Results: The mean age of the patients with Alopecia, other diseases, and healthy population were 9.27, 8.43 , and 9.93 years, respectively. The total difficulty score was not significantly different between the alopecia patients and patients with other diseases $(p=0.137)$. The total difficulty score was significantly different among those with alopecia areata and those with other diseases and the healthy population $(p=0.003$ and $p=0.045)$. Emotional symptoms of conduct problems and peer relationship problems were common in children with alopecia areata compared to the healthy population $(p=0.004, p=0.019$, and $p<$ 0.001 , respectively).

Conclusions: The pediatric patients with alopecia areata have an increased level of behavioral problems compared to the healthy population. Therefore, pediatric patients with alopecia areata need psychiatric assessments alongside treating their dermatologic concerns.

\section{Key Summary Bullet Points}

- behavioral problems of the pediatric population have not been widely studied

- Emotional symptoms, conduct problems, and peer relationship problems were common in children with alopecia areata in comparison with the healthy population

- The pediatric patients with alopecia areata have an increased level of behavioral problems in comparison with the healthy population

\section{Background}

Alopecia areata is an autoimmune disease affecting both children and adults (1). Although the disease is primarily benign; however, younger age of onset is correlated with poor prognosis (2). The previous study from Iran reported that the mean age of onset of alopecia areata in children is 8.9 years, and the scalp is the most common site of involvement (2). Although autoimmune mechanisms have been proposed for the development of alopecia areata; however, the role of other triggers has been highlighted in the literature (3). Alongside the stress-related antigens, including the Major histocompatibility complex class I chain-related gene and its relationship with the development of autoimmune responses by natural killer 
cells, it has been suggested that patients with alopecia areata may have abnormal stress responses during their social life (3). Some psychological issues, including emotional neglect and loss of family members, are among the major triggers and exacerbating alopecia areata (4). Moreover, patients with alopecia areata are more likely to be misbehaved by non-family members, including friends, teachers, and neighbors (4). Therefore, they may experience more psychological difficulties during their daily life.

Mental health disorders are common in children, including various types of behavioral disorders (5). Such problems reduce the quality of life and education (6). Moreover, children with behavioral problems are blamed many times by their parents and the people around them. Chronic diseases, especially those affecting the individuals' appearance, are among the important risk factors for developing behavioral problems in children (6). It has been demonstrated that adult patients with alopecia areata face considerable psychiatric comorbidities, including mood and anxiety disorders $(7,8)$. However, although the prevalence of alopecia areata has been reported to be higher in the pediatric population than adults, the behavioral problems of pediatric patients with alopecia areata have not been widely studied in the literature $(9,10)$.

Therefore, the present study aimed to compare the behavioral problems in children with alopecia areata with control groups using the Iranian version of the strengths and difficulties questionnaire (SDQ).

\section{Methods}

The present case-control study was carried out on the 90 children chosen by simple sampling and equally enrolled in three study groups; Alopecia areata, another skin disease, and healthy children. According to the Liakopoulou et al. study (11), 30 pediatric patients were considered for each study group considering behavioral problems.

This study took place in 4 different dermatology outpatient clinics in Gorgan (located in the north of Iran) and the Taleghani pediatrics clinic of the Golestan University of Medical Science. From May 2019 to February 2020, every patient aged between 4 and 13 years who were diagnosed to have different types of alopecia areata for more than six months enrolled.

Patients receiving therapy by a psychiatrist and those with trichotillomania were not included in the present study. Two control groups, including healthy pediatric patients and patients with other skin diseases, were enrolled in the present study. The parents of every participant completed the Iranian version of the SDQ. The Farsi version of the SDQ has been validated on the Iranian population (Cronbach's alpha: 0.73 for the parent's version) (11). The SDQ evaluates the emotional and behavioral function and contains 25 items rated on 3 points Likert scale. Emotional symptoms, conduct problems, hyperactivity problems, peer relationship problems, and prosocial behavior are the five subscales evaluated by the SDQ (alpha coefficient of prosocial, hyperactivity, emotional symptoms, conduct problems, and peer problems subscales were $0.7,0.71,0.7,0.63$, and 0.009 respectively) (11). 
The Chi-square test was used to evaluate the relationship between different SDQ domains' scores and study groups. SPSS software (version 16) was used and $P<0.05$ was considered as a statistical significant result.

\section{Results}

Mean and standard deviation of age were $9.27 \pm 2.61,8.43 \pm 2.93$, and $9.93 \pm 2.59$ years in Alopecia areata, another skin disease, and healthy children groups, respectively. $(p>0.05)$. Half of the patients in the alopecia group, $70 \%$ of patients with other diseases, and the healthy groups were male $(p>0.05)$.

The results of the SDQ scores are summarized in Table 1. The total difficulty score was not significantly different between the alopecia areata patients and patients with other diseases $(p=0.137)$. However, the total difficulty score was significantly different between those with alopecia areata and the healthy population $(p=0.003)$ and between those with other diseases and the healthy population $(p=0.045)$. Evaluating the SDQ subscales revealed that although the score of emotional symptoms was not different between patients with alopecia areata and patients with other diseases, the score was significantly different between patients with Alopecia and the healthy population $(p=0.004)$. The emotional symptoms were not significantly different among patients with other diseases and the healthy population $(p=0.092)$. The conduct problem scores were no significant differences among the patients with alopecia areata and those with other diseases $(p=0.650)$, and those with other diseases and healthy populations $(p=0.053)$. However, the conduct problem scores differed significantly between patients with alopecia areata and healthy populations $(p=0.019)$. The peer relationship problems were not significantly different between those with alopecia areata and other diseases $(p=0.060)$. However, the peer relationship problems were significantly different between patients with alopecia areata and the healthy population $(p<0.001)$ and patients with other diseases and the healthy population $(p=0.005)$. 
Table 1

Comparison of disorders between Alopecia Areata and control groups

\begin{tabular}{|c|c|c|c|c|c|c|c|c|}
\hline \multirow{2}{*}{\multicolumn{2}{|c|}{ SDQ domain and scores }} & \multirow{2}{*}{\multicolumn{2}{|c|}{$\begin{array}{l}\text { Alopecia } \\
\text { Areata }\end{array}$}} & \multirow{2}{*}{\multicolumn{2}{|c|}{$\begin{array}{l}\text { Other skin } \\
\text { diseases }\end{array}$}} & \multirow{2}{*}{\multicolumn{2}{|c|}{$\begin{array}{l}\text { Healthy } \\
\text { children }\end{array}$}} & \multirow{3}{*}{$\begin{array}{l}\text { P- } \\
\text { value }\end{array}$} \\
\hline & & & & & & & & \\
\hline & & N. & $\%$ & N. & $\%$ & $\mathrm{~N}$ & $\%$ & \\
\hline \multirow[t]{3}{*}{ Emotional symptoms } & Normal (0-3) & 14 & 46.7 & 19 & 63.3 & 26 & 86.7 & \multirow[t]{3}{*}{0.021} \\
\hline & Borderline (4) & 6 & 20 & 5 & 16.7 & 1 & 3.3 & \\
\hline & $\begin{array}{l}\text { Abnormal (5- } \\
\text { 10) }\end{array}$ & 10 & 33.3 & 6 & 20 & 3 & 10 & \\
\hline \multirow[t]{3}{*}{ Conduct problems } & Normal (0-2) & 13 & 43.3 & 14 & 46.7 & 23 & 76.7 & \multirow[t]{3}{*}{0.047} \\
\hline & Borderline (3) & 9 & 30 & 6 & 20 & 2 & 6.7 & \\
\hline & $\begin{array}{l}\text { Abnormal (4- } \\
\text { 10) }\end{array}$ & 8 & 26.7 & 10 & 33.3 & 5 & 16.7 & \\
\hline \multirow[t]{3}{*}{ Hyperactivity problems } & Normal $(0-5)$ & 21 & 70 & 15 & 50 & 23 & 76.7 & \multirow[t]{3}{*}{0.277} \\
\hline & Border (6) & 3 & 10 & 5 & 16.7 & 3 & 10 & \\
\hline & $\begin{array}{l}\text { Abnormal (7- } \\
10)\end{array}$ & 6 & 20 & 10 & 33.3 & 4 & 13.3 & \\
\hline \multirow{3}{*}{$\begin{array}{l}\text { Peer relationship } \\
\text { problems }\end{array}$} & Normal $(0-2)$ & 4 & 16.7 & 11 & 36 & 22 & 76.7 & \multirow{3}{*}{$\begin{array}{l}< \\
0.001\end{array}$} \\
\hline & Border (3) & 8 & 23.3 & 9 & 30 & 0 & 0 & \\
\hline & $\begin{array}{l}\text { Abnormal (4- } \\
\text { 10) }\end{array}$ & 18 & 60 & 10 & 33.3 & 8 & 23.3 & \\
\hline \multirow[t]{3}{*}{ Total difficulties score } & Normal (0-13) & 11 & 36.7 & 16 & 53.3 & 24 & 80 & \multirow[t]{3}{*}{0.006} \\
\hline & Border (14-16) & 9 & 30 & 3 & 10 & 3 & 10 & \\
\hline & $\begin{array}{l}\text { Abnormal (17- } \\
40)\end{array}$ & 10 & 33.3 & 11 & 36.7 & 3 & 10 & \\
\hline \multirow[t]{3}{*}{ Prosocial behavior } & Normal (6-10) & 24 & 80 & 27 & 90 & 24 & 80 & \multirow[t]{3}{*}{0.275} \\
\hline & Border (5) & 0 & 0 & 3 & 10 & 3 & 10 & \\
\hline & $\begin{array}{l}\text { Abnormal (0- } \\
\text { 4) }\end{array}$ & 6 & 20 & 0 & 0 & 3 & 10 & \\
\hline
\end{tabular}

\section{Discussion}


The present study demonstrated that the behavioral problems are more significant in pediatric patients with alopecia areata than in the healthy population. Emotional symptoms, conduct problems, and peer relationship problems differed between pediatric patients with Alopecia and other pediatric diseases and the healthy population.

Alopecia areata has been long considered a psychosomatic disorder. Various psychiatric disorders, including adjustment, depressive, and anxiety disorders, were reported in patients with alopecia areata (12). The disease has different phenotypes with variable degrees of severity and impact (12). The cosmetic impact of the disease is more negatively affected by the severe forms, including the alopecia totalis (12). It has been reported that these patients are more likely to develop pathologic anxiety (12). Regardless of the severity of the disease negatively affecting the behavior, patients with alopecia areata mostly have obsessive, anxiety, and dependent personality traits (12). Therefore, some researchers believe that such personality traits are responsible for abnormal immune responses (12). Another hypothesis suggests that this dermatologic disorder may share some etiologic factors with psychopathologic disorders of childhood, and it has been suggested that the alopecia areata and ADHD may share common underlying mechanisms. A nationwide study conducted by $\mathrm{Ho}$ et al. revealed that alopecia areata risk was higher among ADHD patients (13).

Moreover, patients not receiving methylphenidate had an insignificant reduced risk of developing alopecia areata compared to those who were receiving the treatment (13). Patients with dermatologic disorders are not the only ones who deal with behavioral and psychopathologic disorders. Similarly, it has been demonstrated that pediatric patients with other disorders, including epilepsy deal, face behavioral problems. Salayev et al. compared the SDQ scores in children with epilepsy and the healthy group. They showed that real difficulties and four difficulties subscales of SDQ (hyperactivity problems, emotional symptoms, peer relationship problems, and conduct problems) were significantly higher in the epilepsy group than in the control group (14). Therefore, we decided to evaluate the behavioral disorders of pediatric patients with alopecia areata with two other groups, including a healthy pediatric population and a pediatric population with any other illnesses. We demonstrated that their behavioral disorders are not significantly different between alopecia areata patient and those with other illnesses while both of these groups had a significantly higher rate of behavioral abnormalities in comparison with a healthy population. Almost all of the available studies evaluated the psychopathologic features of the pediatric population with a healthy population. De Waard-Van der Spek et al. demonstrated that children with alopecia areata do not have increased behavioral problems in comparison with the patients with atopic dermatitis (15). They reported that most of the children with both of these diseases have behavioral scores within the clinical range but have lower social competence in comparison with the healthy pediatric population (15). Similar to our study, Liakopoulou et al. demonstrated that the pediatric population with alopecia areata has an increased level of psychopathologies in contrast to the healthy controls (11). They used child behavior checklist scores and their control group had mild diseases. Based on their results, anxiety, and depression symptoms are the core psychopathologic finding in these patients. 
The most critical issue regarding the correlation between alopecia areata and psychopathologic findings in determining the cause and effect relationship between these findings. Although the available studies suggest an increased prevalence of psychopathologic manifestations in alopecia areata pediatric patients; however, it is still unclear whether the psychopathologic findings are a consequence of alopecia areata or the psychopathologic manifestations induced the development of the Alopecia (16). Ghanizade et al. study demonstrated that adolescents and children with alopecia areata have at least one psychiatric disorder (17). Major depressive disorder was the most common type of psychiatric disorder (50\% of patients), and $35.7 \%$ had obsessive-compulsive disorder (17). Reeve et al. study reported that more than half of the patients (58.3\%) had anxiety disorders, and the number of life events regardless of the positivity or negativity of the event was not significantly different in contrast to the healthy population (18). Vélez-Muñiz et al. demonstrated that $77.6 \%$ and $76.7 \%$ of adult and pediatric patients with Alopecia had decreased quality of life. Moreover, $6.3 \%$ of patients with decreased quality of life had signs of depression (19). Therefore considering psychiatric interventions even in pediatric patients presented with any signs of psychiatric disorders is an important issue that should be addressed in clinical settings.

One of the limitations of the present study was using the parent's version of the SDQ questionnaire. Parents with different educational levels statuses may have a different understanding of the questions. The other limitation was considering various morphologic subtypes of alopecia areata (patchy, totalis, ophiasis, universalis), which could be addressed in further studies.

\section{Conclusions}

Our study suggested that pediatric patients with alopecia areata have an increased level of behavioral problems compared to the control group. In contrast with the healthy group, emotional symptoms, conduct problems, and peer relationship problems were common in children with alopecia areata. Therefore, pediatric patients with alopecia areata need psychiatric assessments alongside treating their dermatologic concerns.

\section{Abbreviations}

SDQ :strengths and difficulties questionnaire

\section{Declarations}

\section{Ethics approval and consent to participate:}

The study was conducted by the Declaration of Helsinki. In addition, the study was ethically reviewed by the Ethics Committee of Golestan University of Medical Sciences and was approved with code IR.GOUMS.REC.1396.321. 
All participants were informed that participation is voluntary and reassured that responses would remain confidential. Informed written consent was also obtained from a parent and/or legal guardian for study participation. Participants may withdraw from the trial at any point without any penalty and will not receive compensation for taking part.In the study, personal information about participants collected during the consent/data collection processes are stored securely

\section{Consent for publication:}

Not applicable

\section{Availability of data and materials:}

The datasets used and/or analysed during the current study are available from the corresponding author on reasonable request

\section{Competing interests:}

No potential Competing interests was reported by the authors.

\section{Funding:}

no funding

\section{Authors' contributions:}

Firoozeh Derakhshanpour: study concept and design, and writing the article; najmeh shahini: writing the article, data collection, and literature review; zanireh salami and kimia kazemian : data collection, GholamReza Vaghari: statistical analysis

\section{Acknowledgements:}

the authors would like to thank Golestan University of Medical Sciences for their cooperation.

\section{References}

1. Cranwell W, Sinclair R. Common causes of pediatric alopecia. Australian Journal for General Practitioners. 2018;47:692-6. 
2. Farajzadeh S, Rahnama Z, Esfandiarpour I, Tardast A, Hasheminasab S, Damavandi FD, et al. Clinical and demographic profile of childhood alopecia areata in Iran. Journal of Pakistan Association of Dermatologists. 2013;23:20-7.

3. Barahmani N, de Andrade M, Slusser JP, Zhang Q, Duvic M. Major histocompatibility complex class I chain-related gene A polymorphisms and extended haplotypes are associated with familial alopecia areata. The Journal of investigative dermatology. 2006;126(1):74-8.

4. Taheri R, Behnam B, Alavy Toussy J, Azizzade M, Sheikhvatan M. Triggering Role of Stressful Life Events in Patients with Alopecia Areata. Acta dermatovenerologica Croatica : ADC. 2012;20:246-50.

5. Ogundele MO. Behavioural and emotional disorders in childhood: A brief overview for paediatricians. World journal of clinical pediatrics. 2018;7(1):9-26.

6. Gortmaker SL, Walker DK, Weitzman M, Sobol AM. Chronic conditions, socioeconomic risks, and behavioral problems in children and adolescents. Pediatrics. 1990;85(3):267-76.

7. Ruiz-Doblado S, Carrizosa A, García-Hernández MJ. Alopecia areata: psychiatric comorbidity and adjustment to illness. International journal of dermatology. 2003;42(6):434-7.

8. Alfani S, Antinone V, Mozzetta A, Di Pietro C, Mazzanti C, Stella P, et al. Psychological status of patients with alopecia areata. Acta dermato-venereologica. 2012;92(3):304-6.

9. Alshahrani AA, Al-Tuwaijri R, Abuoliat ZA, Alyabsi M, AlJasser MI, Alkhodair R. Prevalence and Clinical Characteristics of Alopecia Areata at a Tertiary Care Center in Saudi Arabia. Dermatology Research and Practice. 2020;2020:7194270.

10. Lee HH, Gwillim E, Patel KR, Hua T, Rastogi S, Ibler E, et al. Epidemiology of alopecia areata, ophiasis, totalis, and universalis: A systematic review and meta-analysis. Journal of the American Academy of Dermatology. 2020;82(3):675-82.

11. Ahmad G, Ahad I, Gholamreza A. Scale Validation of the Strengths and Difficulties Questionnaire in Iranian Children. Iranian Journal of Psychiatry. 1970;2(2).

12. Ruiz-Doblado S, Carrizosa A, García-Hernández MJ. Alopecia areata: psychiatric comorbidity and adjustment to illness. International journal of dermatology. 2003;42(6):434-7.

13. Ho H-Y, Wong C-K, Wu S-Y, Hsiao RC, Chen Y-L, Yen C-F. Increased Alopecia Areata Risk in Children with Attention-Deficit/Hyperactivity Disorder and the Impact of Methylphenidate Use: A Nationwide Population-Based Cohort Study. International Journal of Environmental Research and Public Health. 2021;18(3):1286.

14. Salayev KA, Sanne B, Salayev R. Psychiatric and behavioural problems in children and adolescents with epilepsy. East Asian Archives of Psychiatry. 2017;27(3):106-14.

15. De Waard-Van der Spek FB, De Raeymaecker DMJ, Root HM, Oranje AP. Alopecia areata and stress in children. Journal of the European Academy of Dermatology and Venereology. 1994;3(1):16-21.

16. Liakopoulou M, Alifieraki T, Katideniou A, Kakourou T, Tselalidou E, Tsiantis J, et al. Children with alopecia areata: psychiatric symptomatology and life events. Journal of the American Academy of Child \& Adolescent Psychiatry. 1997;36(5):678-84. 
17. Ghanizadeh A. Comorbidity of psychiatric disorders in children and adolescents with alopecia areata in a child and adolescent psychiatry clinical sample. International journal of dermatology. 2008;47(11):1118-20.

18. Reeve EA, Savage TA, Bernstein GA. Psychiatric Diagnoses in Children with Alopecia Areata. Journal of the American Academy of Child \& Adolescent Psychiatry. 1996;35(11):1518-22.

19. Vélez-Muñiz RdC, Peralta-Pedrero ML, Jurado-Santa Cruz F, Morales-Sánchez MA. Psychological Profile and Quality of Life of Patients with Alopecia Areata. Skin Appendage Disorders. 2019;5(5):293-8. 